\title{
Tendencias bibliométricas de los resúmenes compilados en las memorias ACFO desde 2010-2017
}

\author{
Bibliometric trends of the abstracts published in Colombian National Meeting of \\ Dental Research from 2010 to 2017
}

\author{
Andrea Johana Almario-Barrera ${ }^{1}$ (D), Sonia Constanza Concha-Sánchez ${ }^{2}$ (D), Álvaro Andrés Angarita ${ }^{3}$, \\ Jessica Vanessa Barón ${ }^{3}$, Juan Pablo Sampayo ${ }^{3}$, Lisset Patricia Bueno ${ }^{3}$, Julieth Paola Arguello³, Daniela Fernanda Álvarez ${ }^{3}$, \\ Laura Juliana Ardila ${ }^{3}$
}

Citación: Almario-Barrera AJ, Concha-Sánchez SC, Angarita AA, Barón JV, Sampayo JP, Bueno LP, Arguello JP, Álvarez DF, Ardila LJ. Tendencias bibliométricas de los resúmenes compilados en las memorias ACFO desde 2010-2017. Ustasalud 2019;18: 17-27.

Licencia Creative Commons

\section{(c) (1) ()요} lo tanto, los lectores pueden acceder libremente a los artículos en su formato .pdf, igualmente podrán descargarlos y difundirlos; sin embargo no podrán modificarlos o alterarlos, adicionalmente se debe reconocer la autoría de las personas que figuran en las publicaciones, pero estas no podrán ser comercializadas

\section{Resumen}

Objetivo: Identificar las tendencias bibliométricas de los resúmenes publicados en las memorias del Encuentro Nacional de Investigación Odontológica (ENIO) de 2010 a 2017. Materiales y métodos: Se realizó un análisis bibliométrico con todos los resúmenes compilados en las memorias mencionadas en el periodo de interés, excluyendo los trabajos presentados por el área de servicios, de estas se extrajo la información relacionada con el evento, indicadores de actividad y colaboración; esta información se sistematizó y procesó en Excel; se calcularon medidas de resumen según la naturaleza de las variables, que se presentaron en textos, tablas y gráficos; las adunas se obtuvieron mediante las aplicaciones VantagePoint y VOSViewer. Resultados: Se identificaron 1135 resúmenes, la mayor proporción estaban registrados en el área clínica (43\%). El 2,8\% de los trabajos provenían de instituciones internacionales, el $47,9 \%$ de instituciones ubicadas en Bogotá, y el $0,8 \%$ eran proyectos multicéntricos. El $11,2 \%$ de las investigaciones son de la Universidad El Bosque, el 10,7\% de la Universidad Nacional de Colombia, el 10,1\% de la Institución Universitaria Colegios de Colombia; la Universidad Santo Tomás aportó el 5,6\%. Predominaron los estudios observacionales (48\%) y estudios In vitro (24,9\%). La aduna muestra que los nodos con mayor número de autores los registra la Universidad El Bosque y con el menor la Universidad Nacional; al referir instituciones, predomina la endogamia, pocos nodos muestran relación entre diferentes universidades. Conclusión: Se encontró predominio de la investigación en el área clínica, especialmente en temas de Patología, Rehabilitación Oral y Ortodoncia. En lo metodológico predominan los estudios epidemiológicos de corte transversal. Se evidenciaron redes de colaboración débiles entre las facultades de odontología del país.

Palabras clave: Bibliometría, investigación, odontología.

\begin{abstract}
Objective: To identify the bibliometric trends of the abstracts published in the Colombian National Meeting of Dental Research memoirs from 2010 to 2017. Methods: A bibliometric analysis was conducted in the period of interest, excluding the projects presented by the services area, from which the information related to the activity indicators was extracted (production, collaboration, and thematic relation); this information was systematized and processed in Excel; summary measures were calculated according to the nature of the variables, which were presented in texts, tables and graphs; the adunas were obtained through the VantagePoint and VOSViewer applications, allowing to visualize the production relation between institutions, authors and thematic axes that guide the research of the different faculties. Results: 1135 compiled abstracts were identified in the analyzed memoirs. According to the area, the highest proportion were registered in clinical area (43.0\%). The $2.8 \%$ of the researches came from international institutions, $47.9 \%$ from institutions located in Bogotá, and $0.8 \%$ were multi-center projects. The $11.2 \%$ of the investigations were carried out by Universidad El Bosque, $10.7 \%$ by UNAL, $10.1 \%$ by UNICOC, and the $5.6 \%$ by Universidad Santo Tomás. In methodology, the observational studies predominate (48\%), In vitro studies (24.9\%). The "aduna" shows that the nodes with the highest number of authors are registered by Universidad El Bosque and the lowest by Universidad Nacional de Colombia; when referring Institutions, inbreeding predominates, few nodes show relation between different Universities. Conclusion: It was found a predominance of research in the clinical area, emphasizing issues of Pathology, Oral Rehabilitation and Orthodontics. In the methodological predominantly cross-sectional epidemiological studies predominate. Weak networks of collaboration between the faculties of dentistry in the country were evident.
\end{abstract}

Keywords: Bibliometrics, research, dentistry.
1 Odontóloga, docente Facultad de Odontología, Universidad Santo Tomás, Bucaramanga, Colombia.

2 Doctora en Salud Pública, docente Facultad de Odontología, Universidad Santo Tomás, Bucaramanga, Colombia.

3 Estudiante vinculado con el Semillero de Investigación de la Facultad de Odontología, Universidad Santo Tomás, Bucaramanga, Colombia.

Autor de correspondencia: Andrea Johana Almario-Barrera Correo electrónico: andrea.almario@ustabuca.edu.co 


\section{INTRODUCCIÓN}

El IV Estudio Nacional de Salud Bucal (ENSAB IV) registró una reducción en la caries dental, pero un incremento en entidades como la enfermedad periodontal, la fluorosis, y el edentulismo. La profesión odontológica en el país enfrenta grandes retos a la hora de responder con calidad, pertinencia y oportunidad a las necesidades bucales de los ciudadanos, $y$ de ofrecer alternativas que respondan a los problemas del sistema estomatognático de los colectivos ${ }^{1}$.

En su práctica profesional, los odontólogos en Colombia tienden a adoptar técnicas, procedimientos y materiales que probaron las casas comerciales $o$ investigadores en otros países, por lo cual su práctica podría estar descontextualizada de la realidad social y económica que enfrentan los colombianos. En el campo de las ciencias básicas, podría estar ocurriendo un fenómeno similar, pues los odontólogos tienden a apropiar el conocimiento que se genera en este campo, a partir de las pesquisas que se realizan en países con mayor desarrollo científico y tecnológico ${ }^{2}$. Muchas de las posibles falencias que enfrenta la profesión odontológica colombiana en el campo de la salud pública, de la clínica, y de las ciencias básicas podrían resolverse mediante la implementación de una investigación contextualizada, pertinente, oportuna y de calidad ${ }^{3}$.

Con todo, cabe señalar que la investigación odontológica en Colombia no es una actividad nueva en el marco de la profesión, pues la Asociación Colombiana de Facultades de Odontología (ACFO) ha incentivado el desarrollo de esta, desde los años noventa y en las diferentes facultades se identifican investigaciones e investigadores que se proponen innovar en la práctica odontológica del país, de ofrecer alternativas para desarrollar un ejercicio ajustado al contexto colombiano, pero también, de responder a las necesidades que en materia de salud pública registran los colectivos en el nivel nacional, y porque no en el internacional. $\mathrm{Mu}$ chas de estas investigaciones se han presentado en los diferentes encuentros de investigación que año a año, desde 1991 realiza la ACFO; sin embargo, muchos de estos trabajos no logran el impacto esperado, al punto que muchos odontólogos e investigadores no las conocen, por lo que se replican de manera innecesaria o sin considerar sus resultados. Por tal motivo, los esfuerzos económicos, sociales y personales que hacen los investigadores, las facultades, y el país no se aprovechan al máximo, lo que representa grandes pérdidas para las personas, la profesión y el Estado ${ }^{2,3}$.

El asesor del área estratégica de investigación de la ACFO recopila y edita los trabajos que año a año presentan los investigadores de las diferentes facultades en todas las áreas de la profesión, como un elemento que orienta al gremio odontológico en la dinámica que vienen desarrollando los investigadores en el país. Pero a pesar de que existen las memorias de las investigaciones realizadas, y que para cada encuentro se hacían las relatorías de las investigaciones presentadas en cada una de las áreas, estos documentos no siempre son accesibles al público en general, adicionalmente, estas relatorías se presentaban como síntesis cualitativas de las investigaciones involucradas, no buscaban analizar las tendencias bibliométricas en los trabajos presentados.

De manera general, la investigación bibliométrica se define como la aplicación de las matemáticas y los métodos estadísticos para describir y comprender los procesos de comunicación escrita, la naturaleza y desarrollo de las disciplinas científicas mediante el recuento y el análisis de esta comunicación, lo que resulta importante, ya que posibilita la obtención de indicadores confiables, de calidad, y con la información que se recolecta de diferentes documentos publicados se reconocen las tendencias de las publicaciones en un campo de la cien$\mathrm{cia}^{4}$. Okubo por su parte, señala que son numerosos los parámetros que se pueden emplear en los análisis bibliométricos; sin embargo, cuando refiere los principales indicadores bibliométricos y su aplicación enfatiza en dos grandes grupos: los primeros relacionados con la actividad de la ciencia y los segundos concernientes a los indicadores de relación ${ }^{5}$. Con base en estos argumentos, el objetivo del presente trabajo fue identificar las tendencias bibliométricas de los resúmenes de investigación publicados en las memorias del Encuentro Nacional de Investigación Odontológica (ENIO), organizado por la Asociación Colombiana de Facultades de Odontología (ACFO) en el periodo de 2010 a 2017.

\section{MATERIALES Y MÉTODOS}

Se realizó un estudio bibliométrico que involucró la totalidad de los resúmenes registrados en las memorias de los Encuentros Nacionales de Investigación Odontológica (ENIO) organizados por la Asociación 
Colombiana de Facultades de Odontología (ACFO), publicados entre 2010 y 2017; se excluyeron los trabajos presentados por el área de servicios.

Las variables incluidas en este trabajo se catalogaron como: Variables relacionadas con el evento (número del evento, año de participación, universidad que organiza, ciudad en la que se realizó). Variables relativas a la actividad científica (modalidad de presentación, área de participación, autores, número de autores, filiación institucional, ciudad de procedencia de los autores, palabras clave y tipo de estudio). Variables relacionadas con indicadores de relación (apellido e iniciales, filiación institucional reseñando la universidad a la que está vinculado el investigador, áreas temáticas, refiriéndolas como salud pública) (cuando abordan colectivos), ciencias básicas (cuando se tratan aspectos relativos a microbiología, bioquímica, genética, entre otros) y clínicas (cuando se involucran aspectos relacionados con técnicas, procedimientos y terapéuticas en el campo profesional propiamente dicho).

Los procedimientos que se llevaron a cabo incluyeron, en primera instancia, recuperar las memorias de los ENIO organizados por la ACFO en el periodo de 2010 a 2017 y, organizar un listado en Excel de la distribución de los resúmenes por año y área de participación. Previó al proceso de extracción de datos se desarrolló un plan de formación de los jóvenes investigadores, en conceptos básicos necesarios para el desarrollo del proyecto, se efectuó la estandarización y se hizo la prueba piloto, momento en el que se verificó el nivel de acuerdo con el proceso de extracción de datos que hacía cada investigador con respecto a una persona con formación avanzada en epidemiología. Se identificaron los potenciales vacíos, se hicieron los correctivos y se procedió a asignar el trabajo correspondiente a cada uno de los jóvenes investigadores, quienes procedieron a sistematizar la información en una base de datos creada en Excel, actividad que fue verificada por el experto en epidemiología de manera continua y para la totalidad de los datos. La información recolectada se procesó en Excel, los datos se organizaron en tablas y gráficos de presentación de resultados.

El plan de análisis estadístico incluyó el cálculo de medidas de resumen según la naturaleza de los datos. Para las cualitativas se calcularon proporciones y para las cuantitativas medidas de tendencia central (pro- medio y mediana) y de dispersión (rango, varianza y desviación estándar) que se procesaron en Excel. Para la generación de los gráficos de adunas (imágenes que buscan integrar los aspectos de interés analizados para facilitar su comprensión) que mostraban las redes y relaciones con los listados generados se hizo la normalización de los datos y se procesaron mediante la aplicación VantagePoint (herramienta informática que permite la recuperación, integración y procesamiento de datos, así como la obtención de resultados a través de diferentes opciones como gráficos, tablas y otros elementos para el reconocimiento de las redes bibliométricas), y se generaron los gráficos en VOSViewer (software que permite elaborar gráficos de redes bibliométricas) para hacer la identificación de las redes más representativas, relativas a autores, instituciones y ejes temáticos.

$\mathrm{Al}$ considerar que la presente investigación se sustentó en la obtención de los datos, a partir de fuentes secundarias, el proceso desarrollado se amparó en la normatividad establecida por la República de Colombia, relacionada con los derechos de autor: En primera instancia, en el artículo 61 de la Constitución Política de Colombia, en el que se establece la protección que el Estado brindará a la propiedad intelectual; adicionalmente, se soporta en la Ley 23 de 1982 sobre los Derechos de Autor. También se soportó en la Ley 599 de 2000 por la cual se emite el Código Penal, en particular en el artículo 170 en el que se puntualiza las sanciones establecidas por la violación de los derechos de autor.

\section{RESULTADOS}

\section{Análisis de variables relacionadas con el evento}

Se identificaron 1135 resúmenes compilados en las memorias analizadas. El 18,9\% se presentó en el ENIO de 2014 organizado por la Facultad de Odontología de la Universidad del Magdalena en Santa Marta. El $17,8 \%$ de los trabajos se divulgaron en el 2017 en el ENIO organizado en la ciudad de Cartagena, por la Facultad de Odontología de la Universidad de Cartagena. El 39,8\% de los trabajos se presentaron en los encuentros nacionales de investigación odontológica realizados por cuatro facultades de Odontología que tienen su sede en Bogotá. Se observa un incremento gradual de los trabajos presentados a lo largo de los años, con alguna fluctuación (Tabla 1). 
Tabla 1. Descripción de variables relacionadas con el evento ACFO realizado en el periodo 2010-2017

\begin{tabular}{ccccc}
\hline Año & Encuentro & $\mathbf{n}(\%)$ & Ciudad & Sede evento \\
\hline 2010 & XXI & $85(7,5)$ & Bogotá & UNICOC \\
2011 & XXII & $98(8,6)$ & Cali & U. Santiago de Cali \\
2012 & XXIII & $111(9,8)$ & Bogotá & UNAL \\
2013 & XXIV & $167(14,7)$ & Pasto & UCC \\
2014 & XXV & $216(19,0)$ & Santa Marta & U. del Magdalena \\
2015 & XXVI & $111(9,8)$ & Bogotá & U. El Bosque \\
2016 & XXVII & $145(12,8)$ & Bogotá & UAN \\
2017 & XXVIII & $202(17,8)$ & Cartagena & U. de Cartagena \\
\hline Total & & $1135(100,0)$ & --- & -- \\
\hline
\end{tabular}

UNICOC: Institución Universitaria Colegios de Colombia; UNAL: Universidad Nacional de Colombia; UCC: Universidad Cooperativa de Colombia; UAN: Universidad Antonio Nariño.

Variables relacionadas con indicadores de actividad científica y de relación que emergen

El 59\% de los trabajos se presentaron en modalidad póster (Figura 1). El área con mayor frecuencia de participación de los trabajos de investigación registrados en las memorias ACFO 2010-2017 fue "Clínicas" (43\%) y la de menor frecuencia "Educación" $(7,4 \%)$ (Figura 2). Los autores con mayor número de trabajos en su orden fueron: Lafaurie-Villamil GI, Martignon S, vinculadas a la Universidad El Bosque de Bogotá, González F de la Universidad de Cartagena, Estrada JH vinculado a la Universidad Nacional de Colombia y de la Universidad Santo Tomás, Aranzazu Moya GC y Martínez López CA (Figura 3). El promedio de número de autores reportados en los resúmenes registrados en estas memorias fue de 4,4 con una desviación estándar de 2,1 autores.

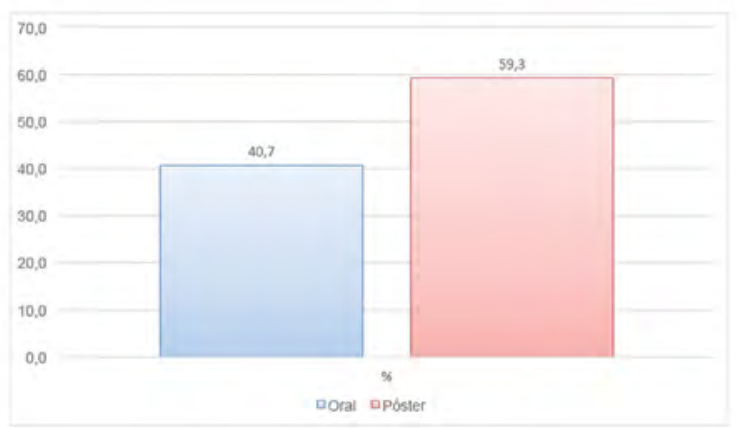

Figura 1. Modalidad de presentación de las investigaciones registradas en las memorias del Encuentro Nacional de Investigación Odontológica organizado por ACFO (2010-2017).

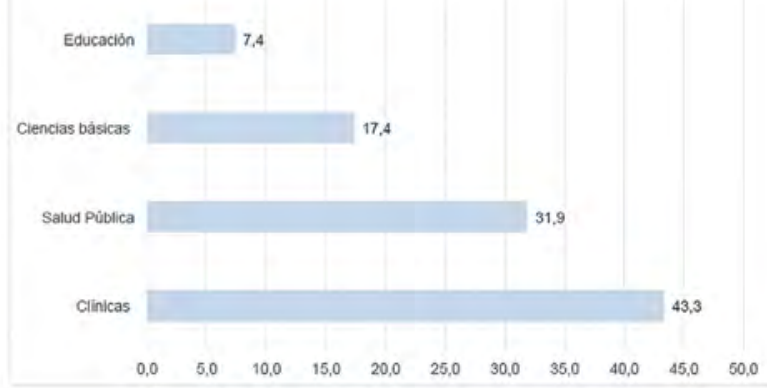

Figura 2. Descripción del área de investigación en la que participó la investigación registrada en las memorias (2010-2017).

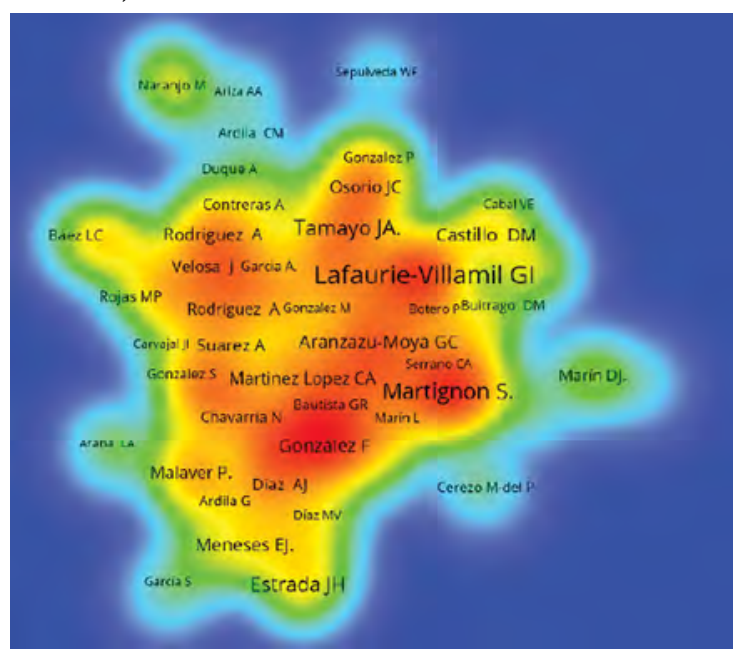

Figura 3. Imagen que permite visualizar la densidad de producción de los autores con investigaciones registradas en las memorias del Encuentro Nacional de Investigación Odontológica (2010-2017). Los autores que se reportan en letras con mayor tamaño son los que tienen mayor densidad de producción. 
Tabla 2. Descripción de la frecuencia de participación de las instituciones en los eventos ACFO realizado en el periodo 2010-2017

\begin{tabular}{lllll}
\hline \multicolumn{1}{c}{ Institución } & \multicolumn{1}{c}{ Ciudad } & n (\%) & Oral & Póster \\
\hline U. El Bosque & Bogotá & $127(11,2)$ & 79 & 48 \\
UNAL & Bogotá & $121(10,7)$ & 58 & 63 \\
\hline UNICOC & Varias sedes & $115(10,1)$ & 37 & 78 \\
\hline U. Cooperativa de Colombia & Varias sedes & $100(8,8)$ & 40 & 60 \\
\hline Pontificia U. Javeriana & Bogotá & $92(8,1)$ & 42 & 50 \\
\hline U. de Cartagena & Cartagena & $87(7,7)$ & 29 & 58 \\
\hline U. del Valle & Cali & $76(6,7)$ & 21 & 55 \\
U. Antonio Nariño & Varias sedes & $66(5,8)$ & 23 & 43 \\
\hline U. Santo Tomás & Bucaramanga & $63(5,6)$ & 20 & 43 \\
\hline U. de Antioquia & Medellín & $47(4,1)$ & 25 & 22 \\
U. Santiago de Cali & Cali & $41(3,6)$ & 12 & 29 \\
FUA de las Américas & Medellín & $37(3,3)$ & 7 & 30 \\
\hline U. Autónoma Manizales & Manizales & $33(2,9)$ & 13 & 20 \\
\hline U. CES & Medellín & $30(2,6)$ & 19 & 11 \\
\hline UNICIEO & Bogotá & $23(2,0)$ & 18 & 5 \\
\hline FU San Martín & Varias sedes & $22(1,9)$ & 7 & 15 \\
\hline U. del Sinú & Varias sedes & $21(1,9)$ & 6 & 15 \\
\hline
\end{tabular}

UNAL: Universidad Nacional de Colombia; UNICOC: Institución Universitaria Colegios de Colombia; FUA de las Américas: Fundación Universitaria Autónoma de Las Américas; FU San Martín: Fundación Universitaria San Martín.

La mayor proporción de trabajos recopilados en las memorias ACFO los presentó la Universidad El Bosque $11,2 \%$, seguido de la Universidad Nacional de Colombia el 10,7\% y el 10,3\% por la Institución Universitaria Colegios de Colombia. La Universidad El Bosque, La Universidad de Antioquia, la Universidad CES y UNI-
CIEO fueron las que presentaron mayor proporción de trabajos en modalidad oral (Tabla 2 y Figura 4 ). El 47,9\% de trabajos provenían de instituciones cuya sede está en Bogotá $(47,9 \%)$ seguido de Cali $(13,6)$, el 2,8\% eran trabajos de instituciones internacionales.

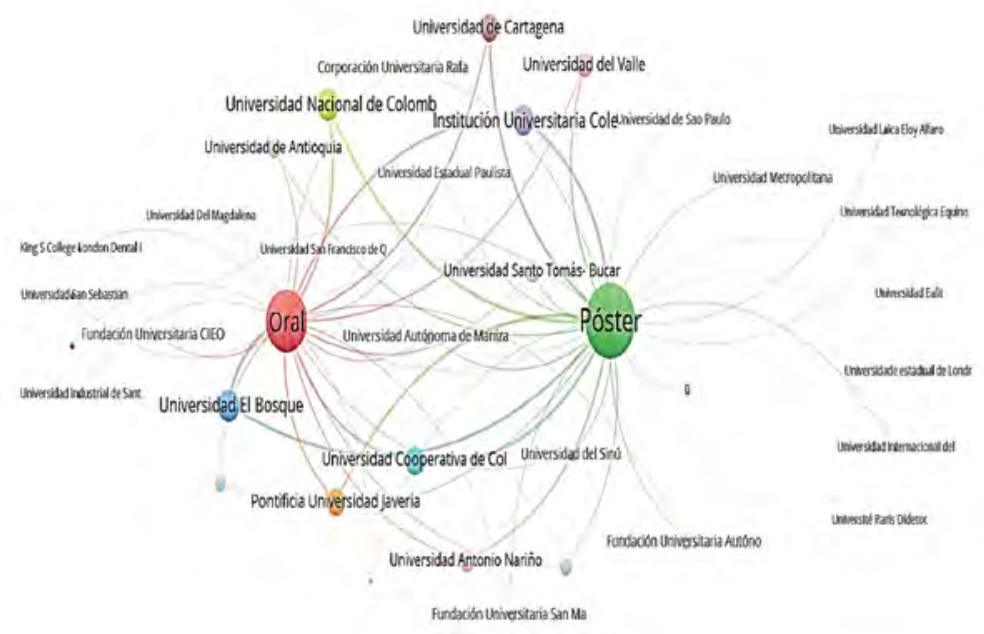

Figura 4. Gráfico que describe la relación de universidades según modalidad de presentación. Las líneas que unen las diferentes instituciones y la modalidad de presentación permiten identificar las redes existentes. 
Al analizar el tipo de estudio predominaron los observacionales (48\%) y los estudios In vitro (24,9\%); había pocas investigaciones con abordaje cualitativo-mixto, revisiones sistemáticas o de evaluación de tecnología diagnóstica $(5,6 \%, 2,4 \%, 2,2 \%$, respectivamente), en el 7,3\% de los resúmenes no se pudo establecer el tipo de estudio (Figura 5).

A partir de las palabras clave se generaron, en VantagePoint, las categorías que agruparon las tendencias de las temáticas abordadas en las investigaciones compiladas en las memorias ACFO 2010-2017 y que se exportaron a la aplicación VOSviewer para la elaboración del gráfico respectivo. En este gráfico de red de palabras se evidencian tres grandes grupos que se representan: en verde, los trabajos relacionados con salud pública, ciclo vital, y educación. En azul, los relativos a aspectos biológicos, microorganismos, patología bucal y factores de riesgo. En rosado, se concentran los trabajos relacionados con terapia, endodoncia, rehabilitación, ortodoncia, esmalte, estadística y procesos analíticos. Estas categorías tienen una estrecha relación con las áreas de salud pública, el grupo de categorías registradas en verde, ciencias básicas, en azul, y clínicas, en rosado (Figura 6). La Figura 7 permite visualizar la red de colaboración entre instituciones, se muestra que son muy pocas las relaciones de colaboración en investigación entre estas.

Cuando se analizan las redes de autores y su relación con el área de participación se evidencia que ciertos investigadores están vinculados a Ciencias Básicas (CB), otros en clínicas (CL), en Salud Pública (SP) o en Educación (ED), estas dos últimas, de acuerdo con el gráfico muy próximas. Las redes permiten visualizar los docentes vinculados a cada una de las áreas de participación, aunque varios investigadores participan en diferentes áreas (Figura 8).

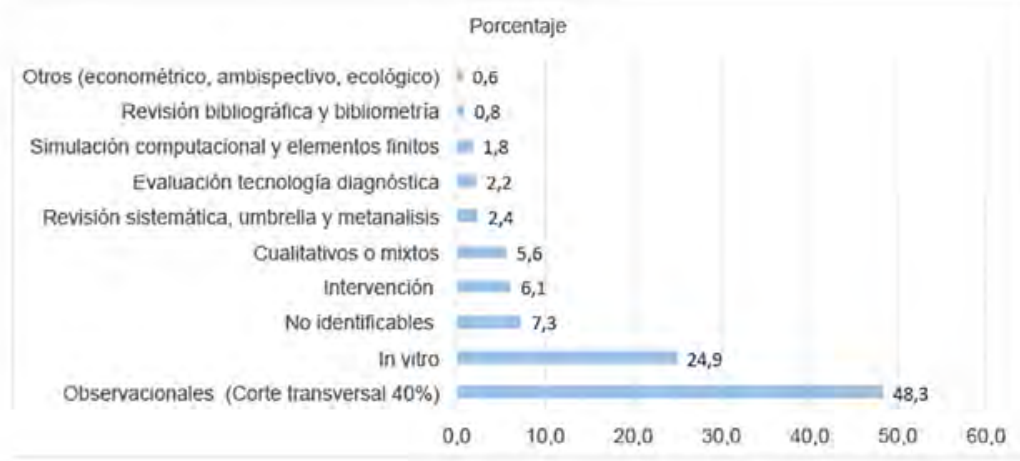

Figura 5. Descripción de los tipos de estudios desarrollados en las investigaciones compilados en las memorias ACFO 2010-2017.

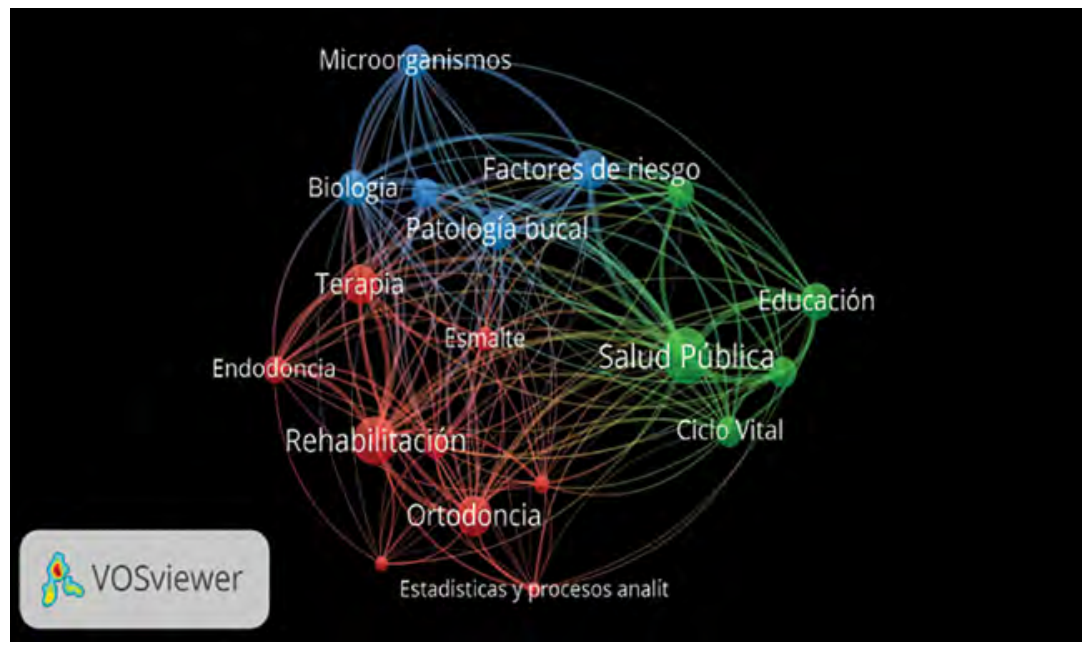

Figura 6. Gráfico que representa las categorías generadas a partir de las palabras clave. Las categorías representadas según color permiten visualizar las relaciones temáticas predominantes. En verde el área de salud pública, rosado clínica y azul ciencias básicas. 


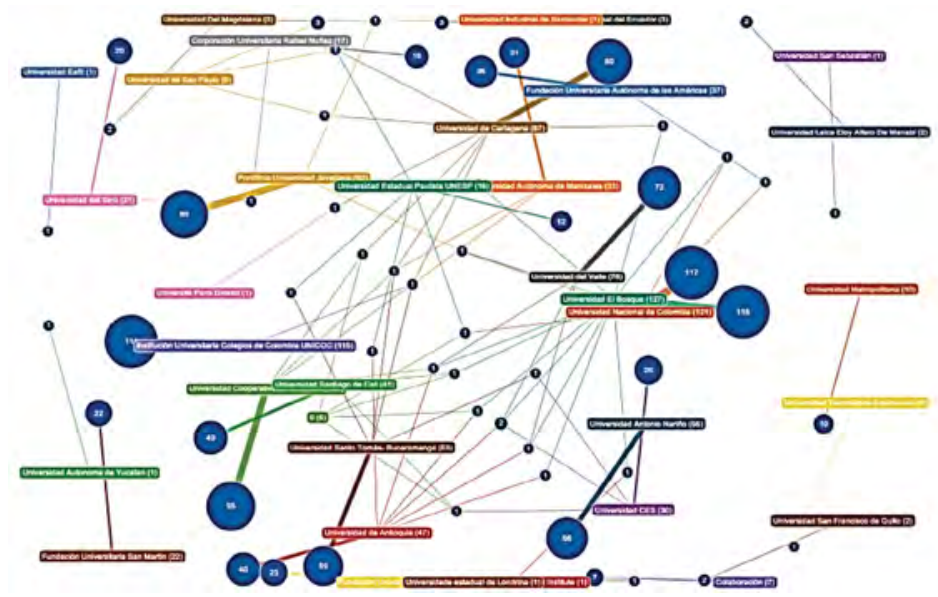

Figura 7. Redes de colaboración entre las diferentes instituciones. Las líneas representan las relaciones de trabajo colectivo entre diferentes instituciones.

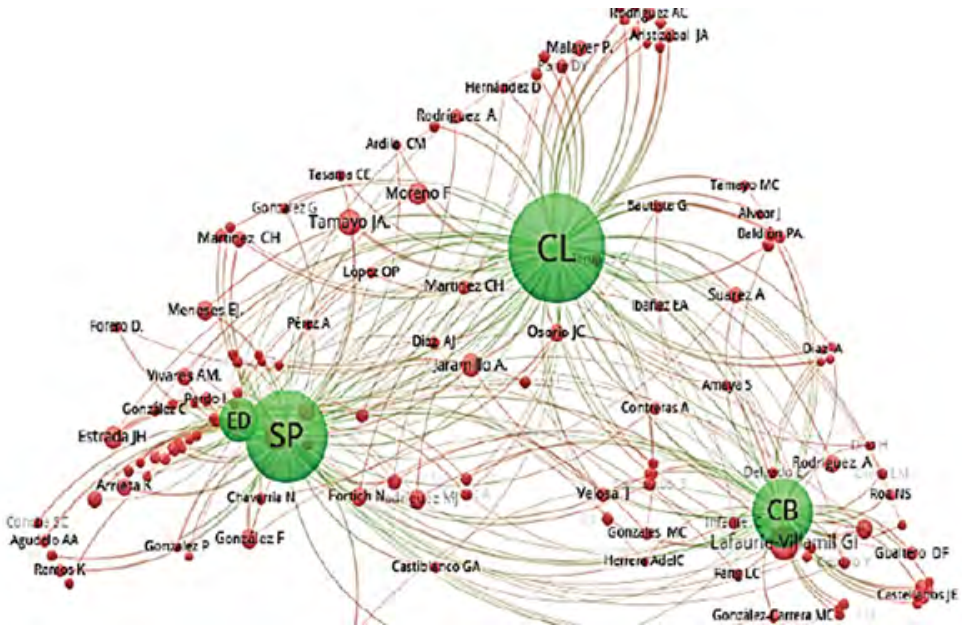

CL: Clínica; SP: Salud Pública; CB: Ciencias Básicas; ED: Educación

Figura 8. Redes de la relación de autores con las áreas de participación; las líneas permiten identificar la proximidad o relación de los autores con los ejes temáticos establecidos.

\section{DISCUSIÓN}

Los hallazgos obtenidos parecen indicar que es muy variable la dinámica de la producción científica, generada por las diferentes facultades de odontología adscritas a la ACFO, que tienen sus resúmenes en estas memorias, y en las que se refleja el proceso en los diversos encuentros de investigación que la Asociación desarrolló en los últimos ocho años.

Cuando se analizan aspectos relacionados con la organización del evento, se evidencia que a lo largo de los años hay un aumento considerable en el número de investigaciones presentadas en estos encuentros, pero el atractivo turístico de lugares como Santa Marta o
Cartagena posiblemente pueden influenciar en la participación de los investigadores en sus encuentros.

Adicionalmente, predominan los trabajos que provienen de facultades cuyas sedes se encuentra en Bogotá, los que se presentan en modalidad póster, las investigaciones que se desarrollan en el área clínica y los estudios descriptivos; son pocas las facultades que concentran la mayor cantidad de trabajos presentados, es baja la participación de instituciones del nivel internacional que muestran sus trabajos en el Encuentro de la ACFO, son muy pocas las redes de colaboración, y a pesar de que si existe preferencia de los investigadores por algún área específica, son numerosos los autores que reportan su participación, tanto en el área de ciencias básicas, en clínica, como en salud pública. 
Comparar estos resultados con otras investigaciones no es un proceso que resulte sencillo, porque los estudios bibliométricos en esta área usualmente analizan la tendencia de la producción científica en alguna revista, en los que comúnmente se visualizan las fortalezas y los alcances de estas publicaciones; así como, el interés que tienen los comités científicos de estas, por conocer la orientación del seriado, su evolución, fortalezas y las posibles limitaciones editoriales que pueden presentar $^{6-18}$. En este sentido, este trabajo que analiza las tendencias bibliométricas de las investigaciones compiladas en las memorias ACFO permiten evidenciar las innovaciones en el área, las temáticas que requieren mayor atención; en relación con esto, cabe señalar que, se evidencian en las memorias trabajos y temáticas innovadoras y nuevos derroteros en las investigaciones presentadas en estos encuentros.

Se evidenció además la importancia de los encuentros como medio de difusión de la investigación odontológica en Colombia, con respecto a esto, se observa un incremento variable en el número de investigaciones e investigadores que participan en estos eventos; para las facultades de odontología los encuentros de investigación siguen siendo una alternativa para interactuar y relacionarse con investigadores en cada uno de los campos de interés.

En cuanto a que existe un buen número de trabajos en los diferentes campos del conocimiento, es importante señalar que la participación en los diferentes eventos incrementa la visibilidad y la accesibilidad de estos trabajos para la comunidad científica nacional; señala también, un aumento progresivo en la participación de los investigadores a estos encuentros, el énfasis que se le da al área clínica, aspecto que parecería ser consistente con la importancia que se le otorga a esta área en la formación profesional del odontólogo; pero también, indican la necesidad de estimular la publicación de trabajos en salud pública, ciencias básicas y, en especial, en el campo de la educación, como también lo señalan Braga y colaboradores; Ferraz y colaboradores; Camps y colaboradores; Yparraguirre-Carbajal y colaboradores ${ }^{7,8,10,12}$.

Jain y colaboradores (2014), en su estudio bibliométrico realizado sobre dos revistas odontológicas que hacen énfasis en salud comunitaria, señalan el predominio de los estudios de tipo descriptivo en las investigaciones publicadas en esas revistas y la necesidad de fortalecer los procesos metodológicos ${ }^{19}$. De otra parte, Navarro y colaboradores analizaron 17 revistas odontológicas, encontrando que el diseño más utilizado fue el corte transversal ${ }^{20}$. En el presente estudio bibliométrico que explora las tendencias en las memorias ACFO 2010-2017 se observó que, igual a lo referido por estos autores, predominaron los estudios de tipo descriptivo, en particular los de corte transversal; adicional a esto, en algunos no se logró identificar el tipo de estudio o no parecen estar bien catalogados. Primo y colaboradores (2014) indican que en los trabajos publicados, en un periodo de diez años en unas revistas de ortodoncia, se percibe un bajo potencial para generar evidencia, ratificando los resultados de los autores ya referidos y los obtenidos en el presente trabajo ${ }^{21}$.

Jiménez Fernández analizó tres décadas de producción científica en el campo odontológico, encontrando un aumento paulatino en la producción en esta área de la ciencia en el periodo analizado ${ }^{22}$; fenómeno que parece similar a lo observado en el análisis de las memorias ACFO 2010-2017, aunque en un periodo más corto. Mishra analizó la producción odontológica entre 2009 y $2014^{23}$; en estos seis años, este investigador de la India encontró un aumento considerable en el potencial de publicación a través de los años, aspectos que parecen coincidir con lo observado en las memorias ACFO publicadas en los últimos ocho años.

Cartes-Velásquez y Aravena-Torres; Bueno-Aguilera y colaboradores; Castro-Rodríguez y Grados-Pomarino; Céspedes; Lara y colaboradores; Silveira y colaboradores, Uribe y colaboradores por su parte exploraron las tendencias de los procesos investigativos en países latinoamericanos mostraron un mayor nivel, estabilidad e impacto cuando hay cooperación internacional, la tendencia a la endogamia en la producción y la poca visibilidad de las revistas colombianas ${ }^{3,24-27}$. Este estudio también se propuso comprender la dinámica de investigación odontológica en Colombia, evidenciándose, igualmente, la endogamia y la baja colaboración con investigadores del ámbito internacional, aspectos que deberían ser fortalecidos en el marco de los Encuentros Nacionales de Investigación Odontológica organizados por la ACFO. Almario y colaboradores (2017) señalan la importancia de fortalecer el trabajo en red de los investigadores en el área 
odontológica, aspecto que parece coincidir con los resultados derivados de la exploración realizada en las memorias del periodo 2010-2017, pues los trabajos en colaboración son muy pocos y las redes parecen débiles ${ }^{30}$.

Al retomar el análisis bibliométrico del IV Encuentro Iberolatinoamericano de Estudiantes de Odontología realizado por Corrales-Reyes y colaboradores en 2017, se observan ciertas similitudes con el proceso de investigación de este trabajo, dado que muestra la potencialidad de evaluar la actividad científica en el marco de un encuentro de investigación, intentando comprender la dinámica del proceso ${ }^{31}$. Si bien la evaluación de Corrales-Reyes y colaboradores se concentró en las memorias de un solo evento, en trabajos desarrollados por estudiantes de odontología y que se publicaron en texto completo ${ }^{31}$, mientras que en este análisis bibliométrico se compilan la actividad de ocho ENIO, de resúmenes de trabajos, presentados por docentes, estudiantes de pregrado y posgrado, así como de semilleros de investigación. En estos dos procesos se evidencia un marcado interés por el área clínica, y pocas redes de colaboración interinstitucional, tanto a nivel nacional como internacional.

El análisis de las investigaciones compiladas en las memorias de los ENIO de los últimos ocho años permitió reconocer la dirección que toma la investigación desarrollada por las facultades de odontología vinculadas con la ACFO en la segunda década del presente siglo, el interés clínico y la necesidad de fortalecer las áreas de salud pública, ciencias básicas y educación; así como la posibilidad de promover las capacidades científicas de la comunidad académica que participa en los diferentes eventos. Los Encuentros Nacionales de Investigación Odontológica organizados por la ACFO parecen ser percibidos por esta comunidad como una oportunidad para fortalecer las competencias investigativas, pero no se visualiza, este espacio, como una opción para fortalecer la integración, la conformación de redes más sólidas e integradas.

Cabría señalar como fortalezas del presente trabajo, el hecho de haber involucrado jóvenes investigadores vinculados al semillero de investigación de la Universidad Santo Tomás, al incentivar con ello sus competencias investigativas. Cabe también resaltar el proceso teórico-práctico que se llevó a cabo en el pro- ceso de estandarización de estos jóvenes adscritos al semillero y a este proyecto que recibió el apoyo financiero de la Universidad Santo Tomás.

El hecho de haber realizado este trabajo soportado en resúmenes y no en investigaciones, en texto completo podría considerarse como una potencial limitación; considerando que, de algunos resúmenes no se pudo extraer toda la información de interés, sin embargo, esta potencial limitación se intentó controlar en el proceso de extracción de datos por parte de los estudiantes, momento en que un docente con alta formación en epidemiología acompañó a los responsables, intentado aclarar las dudas y proporcionando validez al proceso desarrollado. Con base en este proceso investigativo se concluye que:

- Cuando se analizan los aspectos relativos a la producción científica se evidencia que en los trabajos compilados en las memorias ACFO hay mayor interés de investigar en las áreas clínicas, en particular en aspectos relacionados con patología, rehabilitación oral y ortodoncia; seguido de temáticas relativas a la salud pública y ciencias básicas; evidenciándose la necesidad de fortalecer aspectos asociados a la educación en el campo odontológico, en este sentido, se observa la importancia de una formación integral del odontólogo que va más allá de lo clínico y lo puramente profesional.

- En cuanto a los aspectos relacionados con la filiación institucional y de las relaciones de los investigadores se percibe que las facultades de odontología de la Universidad El Bosque, la Universidad Nacional de Colombia y de la Institución Universitaria Colegios de Colombia parecen concentrar un buen número de los trabajos publicados en las memorias ACFO; se observan redes de trabajo débiles que requieren ser fortalecidas y promover el desarrollo de trabajos con instituciones del nivel internacional en las investigaciones compiladas en estas memorias. En el campo de la investigación se señala que las relaciones entre investigadores es un pilar fundamental en el desarrollo, no solo de la investigación, sino muy especialmente de la profesión odontológica.
ÜSTASTALALUD 
La producción científica de los trabajos publicados en las memorias de la ACFO de los últimos ocho años y que representan la dinámica de la investigación de la segunda década del siglo XXI muestra un incremento gradual a lo largo de los años con algunas fluctuaciones. En cuanto a la metodología cabe señalar que predominaron los estudios observacionales de corte transversal, que se podrían catalogar como estudios que proveen bajo nivel de evidencia, señalando el predominio de la investigación desde perspectivas positivistas y la necesidad de promover las competencias de la investigación odontológica en Colombia.

\section{Agradecimientos}

A los estudiantes del espacio académico Semilleros de Investigación I y Semillero de Investigación II que participaron en la recolección de los datos.

\section{REFERENCIAS}

1. Ministerio de Salud y Protección Social de Colombia. IV Estudio Nacional de Salud Bucal (ENSAB IV). Bogotá; 2015.

2. Concha SC, Pieschacón MP, Rodríguez MJ. Caracterización de los grupos de investigación en el área odontológica registrados en Colciencias a diciembre de 2012. Rev. Fac Odontol Univ Antioq 2014;25(2):233-4.

3. Lara N, López V, Mendoza S. La investigación odontológica en México (2001-2008). Revista ADM. 2011;68(5):229-236.

4. Rincón ML. Discurso en homenaje a su nombramiento como presidenta de la Asociación Colombiana de Facultades de Odontología [Editorial]. Ustasalud. 2009;8(1):5-6.

5. Álzate Piedrahita MV, Gómez Arbelaez C, Gómez Mendoza MA, Loaiza Romero F. Bibliometría y discurso pedagógico: un estudio de la Facultad de Ciencias de la Educación de la Universidad Tecnológica de Pereira U.T.P. [Internet]. Pereira: Universidad Tecnologica de Pereira; 2004 [citado 2 de enero de 2019]. p. 229. URL disponible en: http://books. google.com.co/books?id=0Q-MMwEACAAJ

6. Okubo Y. Bibliometric Indicators and Analysis of Research Systems: Methods and Examples. OECD Science. 1997 [citado 20 de enero de 2019];1. URL disponible en: https://www.oecd-ilibrary.org/science-and-technology/ bibliometric-indicators-and-analysis-of-research-systems_208277770603. DOI: 10.1787/208277770603.
7. Braga P, Ferreira SH, Poletto C, Bervian V, Kramer F. Bibliometric evaluation of the scientific production of the Stomatos Dental Journal. Stomatos. 2011;17(33):20-31.

8. Ferraz VCT, Amadei JRP, Santos CF. The evolution of the journal of applied oral science: a bibliometric analysis. J Appl Oral Sci. 2008;16(6):420-7. DOI: 10.1590/S1678-77572008000600012.

9. Rodríguez MJ, Pieruccini JF, Pieruccini SP, Concha SC. Tendencias de publicación en Ustasalud desde 2002 hasta 2014: Un análisis bibliométrico. Ustasalud; 13:40-8. DOI: 10.15332/us.v13i1.1397.

10. Camps D, Recuero Y, Samar ME, Avila RE. Análisis bibliométrico de tesis de doctorado del área de las Ciencias de la Salud: primera parte. Odontología. 2005; - E-LIS repository [Internet]. [citado 4 de febrero de 2019]. URL disponible en: http://eprints.rclis.org/7783/

11. Boric V. Bibliometric analysis of the articles from the School of Dental Medical University of Zagreb, indexed in Web of Science database (1976-2005). Acta Stomatol Croat. 2006;40(3):218-35.

12. Yparraguirre-Carbajal J, Telles-Mimbela P, Borja-Guerrero P, Alfaro-Carballido D. Estudio bibliométrico de los artículos publicados en la revista odontológica de una universidad peruana. Kiru. 2013;10(1):32-7.

13. Cartes-Velásquez R. Manterola C. Bibliometric analysis of articles in ISI dental journal, 2007-2011. Scientometrics. 2014;98:2223-33.

14. Patrón C, López MdC, Piovesan S, Demaría B. Análisis bibliométrico de la producción científica de la revista Odontoestomatología. Odontoestomatología. 2014;16(23):34-43.

15. Delgado-Troncoso JE. Revistas científicas odontológicas de libre acceso en Iberoamérica y Colombia. Rev. Fac Odontol Univ Antioq. 2014; 26(1):126-51.

16. Restrepo-Valencia L, Cano A, Angarita LD, Castañeda C, Sánchez RD, González-Ariza S. Análisis de la producción científica de la revista CES Odontología en los últimos 10 años. CES Odontol. 2015;28(2):119-131.

17. Corrales IE, Reyes JJ. Fornaris U. Bibliometric analysis of the journal of oral research. Period 2012-2015. J Oral Res 2016: 5(5):188-93. DOI: 10.17126/joralres.2016.042.

18. Castro-Rodríguez YA. Perfil bibliométrico de la producción científica de la revista odontológica Sanmarquina, 2005-2014. Odontol Sanmarquina. 2017; 20(1):13-16. 
19. Jain S, Patthi B, Singla A, Singh S, Singh K, Kundu H. Bibliometric analysis of two journals of community dentistry. Journal of Indian Association of Public Health Dentistry. 2014;12(4):250-60. DOI: 10.4103/2319-5932.147642.

20. Navarro P, Cantín M, Ottone NE. Diseños de investigación utilizados en revistas odontológicas de la red SciELO. Una visión bibliométrica. Avances en Odontoestomatología. 2016;32(3):153-158.

21. Primo NA, Gazzola VB, Primo BT, Tovo MF, Faraco Junior IM. Bibliometric analysis of scientific articles published in Brazilian and international orthodontic journals over a 10-year period. Dental Press J Orthod. 2014;19(2):56-65.

22. Jiménez Fernández I. La actividad científica en odontología. Análisis de las tres últimas décadas a través de web of science. Dialnet [Internet]. 2013;1. [citado 2 de febrero de 2019]. URL disponible en: https://dialnet.unirioja.es/ servlet/tesis? codigo $=58809$

23. Mishra L, Pattanik P, KUmar M, Aggrwal S, Misra SR. A bibliometric analysis of two pubmed-index high impact factor endodontic journals: A comparison of Indian with other countries. Indian Journal of Dentistry. 2016;7(3):1211-125.

24. Cartes-Velásquez R, Aravena Torres P. Perfil bibliométrico de la odontología chilena, 2001-2010. Rev Clin Periodoncia Implantol Rehabil Oral. 2012;5(1):5-8.

25. Bueno-Aguilera F, Jiménez-Contreras E, Lucena-Martín C, Pulgar-Encinas R. Dental research in Spain. A bibliometric analysis on subjects, authors and institutional (1993-2012). Med Oral Patol Oral Cir Bucal. 2016;21(2):142-50. DOI: 10.4317/medoral.20756.
26. Castro-Rodríguez Y, Grados-Pomarino S. Productividad científica de revistas odontológicas peruanas. Evaluación de los últimos 10 años. Educ Med. 2017;18(3):174-178.

27. Céspedes MX. Tendencia de publicación en las revistas odontológicas colombianas indexadas en Publindex: Un análisis bibliométrico [Trabajo de grado]. Bucaramanga: Universidad Santo Tomás; 2016.

28. Silveira C, Casnati B, López MdC. Evolución de las publicaciones científicas en odontología Uruguay 1910-2007. Parte I. Odontoestomatología. 2009;11(12):51-58.

29. Uribe SE, Uribe DS, Schuman WA. Perfil bibliométrico de revistas odontológicas de Chile del periodo 20022012. Rev Clin Periodoncia Implantol Rehabil Oral. 2014;7(2):76-84.

30. Almario-Barrera AJ, Acevedo-Arguello C, Villarreal-Neira C, Morales-Borrero C, Concha-Sánchez SC. Oral health and dental care in pregnant women: Bibliometric study, systematic review, and content analysis. Rev.Fac Odontol Univ Antioq. 2017;28(2):374-407.

31. Corrales-Reyes IE. Reyes-Pérez JJ, Fornaris-Cedeño YF. Análisis bibliométrico del IV Encuentro Iberolatinoamericano de Estudiantes de Odontología. Inv Ed. Med. 2017;6(23):153-159.

\section{Correos electrónicos de los autores:}

Andrea Johana Almario-Barrera: andrea.almario@ustabuca.edu.co

Sonia Constanza Concha-Sánchez: sonia.concha@ustabuca.edu.co

Álvaro Andrés Angarita: alvaro.angarita@ustabuca.edu.co Jessica Vanessa Barón: jessica.baron@ustabuca.edu.co Juan Pablo Sampayo: juan.sampayo@ustabuca.edu.co Lisset Patricia Bueno: lisset.bueno@ustabuca.edu.co Julieth Paola Arguello: julieth.arguello@ustabuca.edu.co

Daniela Fernanda Álvarez: daniela.alvarez@ustabuca.edu.co Laura Juliana Ardila: laura.ardila03@ustabuca.edu.co 